ARTICLE

https://doi.org/10.1038/s41467-019-13581-9

\title{
Strong optical response and light emission from a monolayer molecular crystal
}

Huijuan Zhao 1,11, Yingbo Zhao 2,11, Yinxuan Song 3,11, Ming Zhou (1) 4, Wei Lv', Liu Tao1, Yuzhang Feng ${ }^{5}$, Biying Song ${ }^{5}$, Yue Ma ${ }^{1}$, Junqing Zhang ${ }^{1}$, Jun Xiao ${ }^{6}$, Ying Wang ${ }^{6}$, Der-Hsien Lien², Matin Amani², Hyungjin Kim², Xiaoqing Chen ${ }^{1,7}$, Zhangting $\mathrm{Wu}^{8}$, Zhenhua $\mathrm{Ni}^{8}$, Peng Wang ${ }^{5}{ }^{5}$, Yi Shi (1) ${ }^{1}$, Haibo Ma ${ }^{3 \star}$, Xiang Zhang ${ }^{6}$, Jian-Bin $\mathrm{Xu}^{9}$, Alessandro Troisi ${ }^{10}$, Ali Javey (i) ${ }^{2 \star} \&$ Xinran Wang (i) ${ }^{1 \star}$

Excitons in two-dimensional (2D) materials are tightly bound and exhibit rich physics. So far, the optical excitations in 2D semiconductors are dominated by Wannier-Mott excitons, but molecular systems can host Frenkel excitons (FE) with unique properties. Here, we report a strong optical response in a class of monolayer molecular J-aggregates. The exciton exhibits giant oscillator strength and absorption (over 30\% for monolayer) at resonance, as well as photoluminescence quantum yield in the range of $60-100 \%$. We observe evidence of superradiance (including increased oscillator strength, bathochromic shift, reduced linewidth and lifetime) at room-temperature and more progressively towards low temperature. These unique properties only exist in monolayer owing to the large unscreened dipole interactions and suppression of charge-transfer processes. Finally, we demonstrate light-emitting devices with the monolayer J-aggregate. The intrinsic device speed could be beyond $30 \mathrm{GHz}$, which is promising for next-generation ultrafast on-chip optical communications.

\footnotetext{
${ }^{1}$ National Laboratory of Solid State Microstructures, School of Electronic Science and Engineering, and Collaborative Innovation Center of Advanced Microstructures, Nanjing University, Nanjing 210093, China. ${ }^{2}$ Department of Electrical Engineering and Computer Sciences, University of California at Berkeley, Materials Sciences Division, Lawrence Berkeley National Laboratory, Berkeley, CA 94720, USA. ${ }^{3}$ Key Laboratory of Mesoscopic Chemistry of MOE, School of Chemistry and Chemical Engineering, Nanjing University, Nanjing 210093, China. ${ }^{4}$ Department of Electrical and Computer Engineering, University of Wisconsin, Madison, Madison 53705, USA. ${ }^{5}$ National Laboratory of Solid State Microstructures, Jiangsu Key Laboratory of Artificial Functional Materials, College of Engineering and Applied Sciences and Collaborative Innovation Center of Advanced Microstructures, Nanjing University, Nanjing 210093, China. ${ }^{6}$ NSF Nanoscale Science and Engineering Center (NSEC), University of California, Berkeley, CA 94720, USA. ${ }^{7}$ School of Microelectronics, Xidian University, Xian 710071, China. ${ }^{8}$ Department of Physics, Southeast University, Nanjing 211189, China. ${ }^{9}$ Department of Electronic Engineering, The Chinese University of Hong Kong, Hong Kong 999077, China. ${ }^{10}$ Department of Chemistry, University of Liverpool, Liverpool L69 7ZD, U.K. ${ }^{11}$ These authors contributed equally: Huijuan Zhao, Yingbo Zhao, Yinxuan Song. *email: haibo@nju.edu.cn; ajavey@berkeley.edu; xrwang@nju.edu.cn
} 
T he reduced Coulomb screening at low dimensions has led to many fascinating phenomena in two-dimensional (2D) materials ${ }^{1}$. The exciton binding energy can exceed hundreds of $\mathrm{meV}$, more than one order of magnitude larger than bulk semiconductors and quantum-well structures ${ }^{2,3}$. In addition, the large charge-dipole and dipole-dipole interaction ( tens of meV) can stabilize many-body complexes such as trions and bi-excitons even at room temperature ${ }^{4,5}$. While most studies have focused on atomic crystals, many molecular semiconductors also have $2 \mathrm{D}$ layered form ${ }^{6-10}$. Compared to popular 2D semiconductors such as transition metal dichalcogenides (TMDs), the dipole-dipole interaction is even stronger in molecular systems given the localized nature of excitation (e.g., Frenkel excitons (FE) is localized on a single molecule) and low dielectric constant. Therefore, it is expected that dimensionality control of molecular semiconductors would substantially modify the excitonic coupling and lead to new optoelectronic functions.

A particularly interesting system to study exciton interaction is molecular J-aggregate. First observed in $1930 \mathrm{~s}^{11}$, it is characterized by red-shift and narrowing of spectral lines due to constructive quantum superposition of monomer excitations ${ }^{12}$. These delocalized states are less perturbed by disorders (analogy of Bloch wave in crystalline solids) and therefore highly desirable for improving device functions $s^{13}$. However, in most cases the extent of coherence is rather limited because the energy relaxation is dominated by inter-molecular CT (also called self-trapping) in ultrafast sub-picosecond time scale, among other processes ${ }^{14}$. To this end, $2 \mathrm{D}$ assembly is extremely appealing due to enhanced Coulomb interaction between local Frenkel dipoles and complete suppression of interlayer CT. In fact, the most efficient lightharvesting complexes found in nature, the chlorosome, contains dye molecules organized into 2D lamellar form ${ }^{15}$.

Recently, we have developed vapor-transport-based means to precisely assembled molecules on 2D surfaces, opening the possibility to investigate their electrical and optical properties ${ }^{16,17}$. In this work, we study the optical properties of monolayer (ML) perylene derivatives, namely dimethyl-3,4,9,10-perylenetetracarboxilic diimide (Me-PTCDI), 3,4,9,10-perylene-tetracarboxylic diimide (PTCDI) and 3,4,9,10-perylene-tetracarboxylic dianhydride (PTCDA), where the molecular packing ensures strong dipole interaction but weak electronic coupling, an ideal condition for long-range J-aggregation. We find that the exciton has giant oscillator strength, leading to strong absorption (over 15 and $30 \%$ at room temperature and $4 \mathrm{~K}$, respectively) and bright emission (more than two orders of magnitude brighter than semiconducting TMDs). Combined temperature-dependent experiments and density functional theory (DFT) calculations suggest that the size of exciton wavefunction $\left(N_{c}\right)$ gradually builds up and could reach the order of hundred molecules at low temperature, which is similar to Wannier excitons in inorganic semiconductors. We further fabricate transient light-emitting devices on ML perylene derivatives and show that the excitonic state is robust under electrical excitation. The observed near$100 \%$ photoluminescence quantum yield (PLQY), sub-30 ps radiative lifetime and electrical injection pave the way for high efficiency, high speed light-emitting devices enabled by coherent dipole interactions.

\section{Characterization and room-temperature optical properties of ML Me-PTCDI crystals}

To grow macroscopically high-quality perylene derivatives, we carried out physical vapor transport in a tube furnace using mechanically exfoliated hexagonal boron nitride (h-BN) as substrate $^{18}$ (see "Methods" section). Layered morphology of all three molecular crystals with step height of $\sim 3.3 \AA$ down to ML was revealed by atomic force microscopy (AFM) (Fig. 1b, c, Supplementary Fig. 1), indicating face-on molecular packing. We did not observe any evidence of polymorphism between ML and multi-layer samples. Figure 1a shows the in-plane herringbone lattice structure of Me-PTCDI $(a=21.8 \AA, b=13.6 \AA)$ determined from selected-area electron diffraction (SAED) and confirmed by high-resolution AFM (Supplementary Fig. 2). Our structural characterizations were consistent with previous experiments and molecular dynamics simulations on 2D surfaces ${ }^{19,20}$, but with slightly different lattice parameters from bulk (102) plane ${ }^{21}$ likely due to the interaction with h-BN substrate. Since the three molecules show qualitatively the same behavior, below we focus our discussion on Me-PTCDI (see Supplementary Note 1 for the discussion on PTCDA and PTCDI).

The lack of $\pi-\pi$ stacking in ML results in minimal in-plane electronic coupling and CT (Supplementary Fig. 3, Supplementary Table 1). On the other hand, reduced screening gives strong attractive dipole-dipole coupling $J \sim-23 \mathrm{meV}$ and forms Jaggregation. The large $J$ means that many-body interaction should be significant even at room temperature, leading to interesting layer-dependent optical properties. Figure 1e shows the PL image of a Me-PTCDI sample with both ML and multilayer regions, taken by a color CCD camera under $450 \mathrm{~nm}$ LED illumination (Fig. 1d is the microscope image of the sample). While the multi-layer regions showed bulk-like red luminescence, the ML regions showed distinct green luminescence (this phenomenon was reproduced in all samples). The change of color was not gradual but occurred abruptly at ML (Supplementary Fig. 4). Furthermore, the PL was anisotropic with $180^{\circ}$ period as shown by polarization-resolved PL imaging (Fig. 1g, h). The uniform change of intensity across the whole ML region suggested that it was single crystalline and that the green luminescence was an intrinsic property of ML crystal rather than from defects or impurities. The change of luminescent color was also reflected in PL spectrum, where ML and multi-layer samples peaked near 2.22 and $1.80 \mathrm{eV}$, respectively (Fig. 1f). We also compared the static and time-resolved PL with monomers spin cast on h-BN from very dilute dimethyl sulfoxide solution. We observed simultaneous red shift from monomer 0-0 Frenkel transition (by $\sim 50 \mathrm{meV}$, Fig. 1f), reduced linewidth and vibronic progressions, as well as lifetime shortening by more than one order of magnitude (27 ps vs. 556 ps, Fig. 2), which were typical signs of superradiance in J-aggregate ${ }^{12}$. These experimental evidences suggested exciton delocalization over $N_{\mathrm{c}} \sim 10$ molecules at room temperature, as expected from the large $J$ and further corroborated by DFT calculations (Supplementary Fig. 10).

We measured the room temperature PLQY of ML Me-PTCDI under $2.41 \mathrm{eV}$ excitation ${ }^{22}$, where the absorption was $\sim 2.4 \pm 0.2 \%$ (see "Methods" section). Remarkably, a significant portion of samples showed near-100\% PLQY (Fig. 2a, see Supplementary Fig. 5b for statistics of 19 samples). As reference, the PLQY of multi-layer Me-PTCDI was only $\sim 4.8 \%$ (Fig. 2a), due to the dominant inter-layer CT and $\mathrm{H}$-aggregation effects ${ }^{23,24}$. The low PLQY for multi-layer samples is consistent with thin film ${ }^{23}$. Although single-molecule Me-PTCDI in solution can exhibit 93\% quantum efficiency ${ }^{23}$, the near-100\% PLQY in solid-state aggregate is unprecedented. Importantly, the PLQY of ML was maintained throughout the entire range of pump power without any decay, indicating that higher order non-radiative decay processes such as Auger and biexitonic recombination did not play important roles as in $\mathrm{TMDs}^{22}$. This is a huge advantage in device applications. Currently, even direct-gap TMDs and black phosphorous have low PLQY on the order of $10 \%$ or less $22,25,26$, which posts fundamental limit on the efficiency of light-emitting devices $^{3,27}$. Although chemical treatment can improve the PLQY 
a

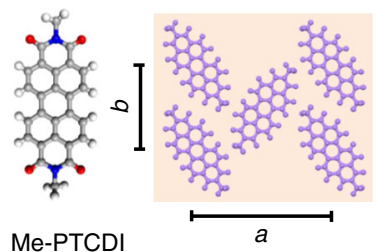

Me-PTCDI b

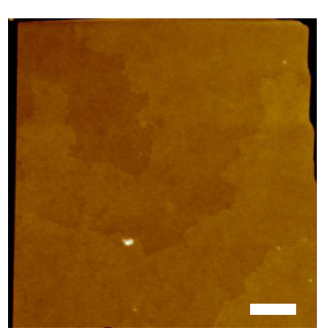

e

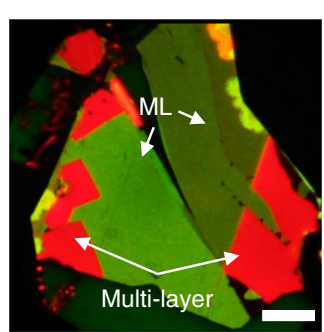

C

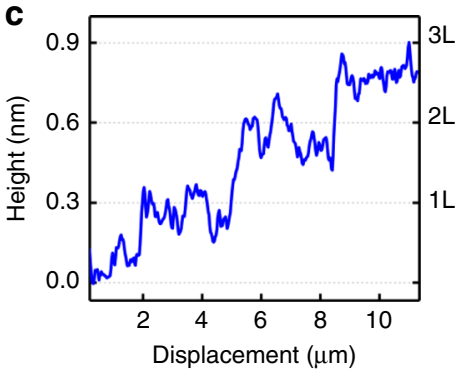

f

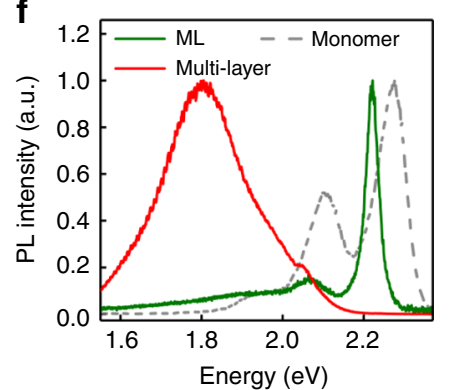

$9_{0}^{\circ}$

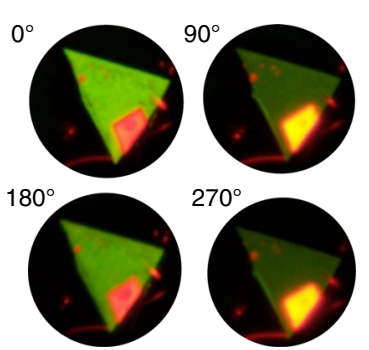

$\mathbf{h}$

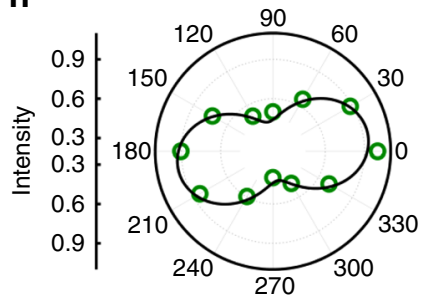

Fig. 1 Structural characterization and thickness-dependent optical properties of 2D Me-PTCDI crystals. a In-plane molecular packing of ML Me-PTCDI on h-BN. b AFM image of a multi-layer Me-PTCDI sample showing the layered morphology. Scale bar: $2 \mu$ m. $\mathbf{c}$ The height profile of Me-PTCDI steps measured from (b). d, e Optical microscope and PL image of a Me-PTCDI sample with both ML and multi-layer regions marked by the white arrows. The different green colors in $\mathrm{ML}$ regions is due to slight variations of the spectrum (such as intensity ratio between the peaks). Scale bars: $10 \mu \mathrm{m}$. $\mathbf{f} \mathrm{PL}$ spectra of ML Me-PTCDI, multi-layer and monomer at room temperature. $\mathbf{g}$ Polarization-resolved PL images of a ML Me-PTCDI samples at four different rotation angles. The uniform color change proves single crystalline nature of the ML. h Normalized PL intensity collected from ML region in (g).

a

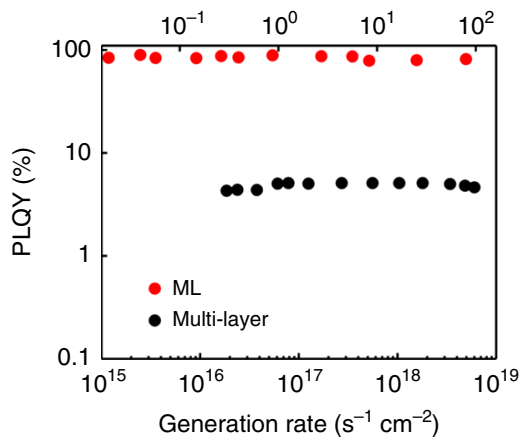

b

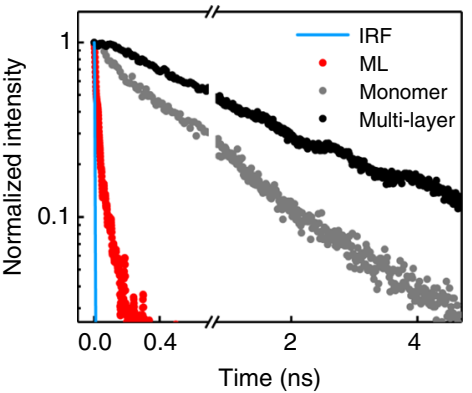

C

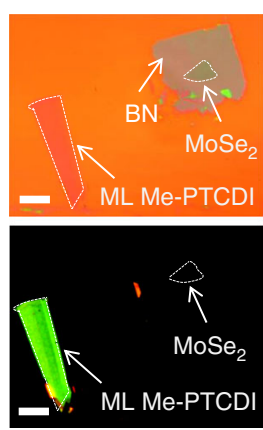

Fig. 2 Thickness-dependent PLQY and lifetime at room temperature. a PLQY of a ML and multi-layer Me-PTCDI as a function of pump power (optical generation rate). $\mathbf{b}$ PL lifetime of Me-PTCDI ML, multi-layer and spin cast monomer, measured with streak camera (the instrument response function (IRF) is shown as reference). The ML data can be fitted with two components. The faster component $(\tau \sim 27 \mathrm{ps})$ is attributed to radiative recombination because of the high PLQY. The slower component ( $\tau \sim 200 \mathrm{ps}$ ) could be non-radiative due to traps or disorder, which only contributes to $7.5 \%$ of the total excitation. The multi-layer and monomer data can be fitted with a single component with $\tau \sim 1.73 \mathrm{~ns}$ and $556 \mathrm{ps}$, respectively. c Room-temperature optical microscope (top) and PL image (bottom, under $450 \mathrm{~nm}$ excitation) of a ML Me-PTCDI and ML MoSe 2 sample, both on h-BN. While the Me-PTCDI shows bright luminescence, the $\mathrm{MoSe}_{2}$ is nearly invisible. Scale bars: $10 \mu \mathrm{m}$. 

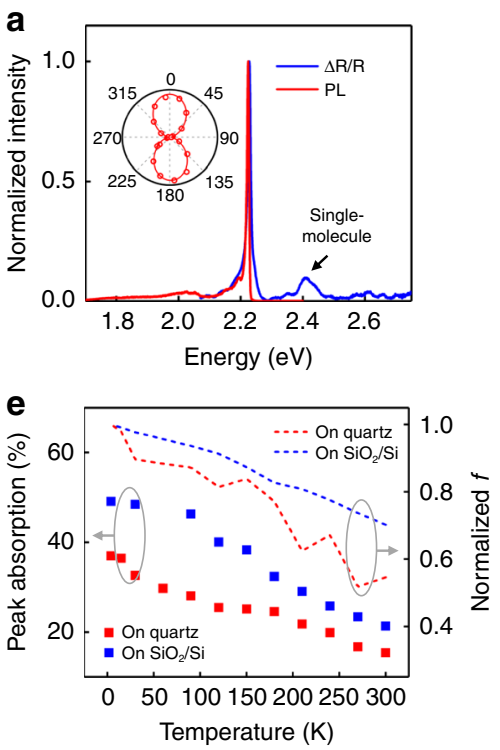
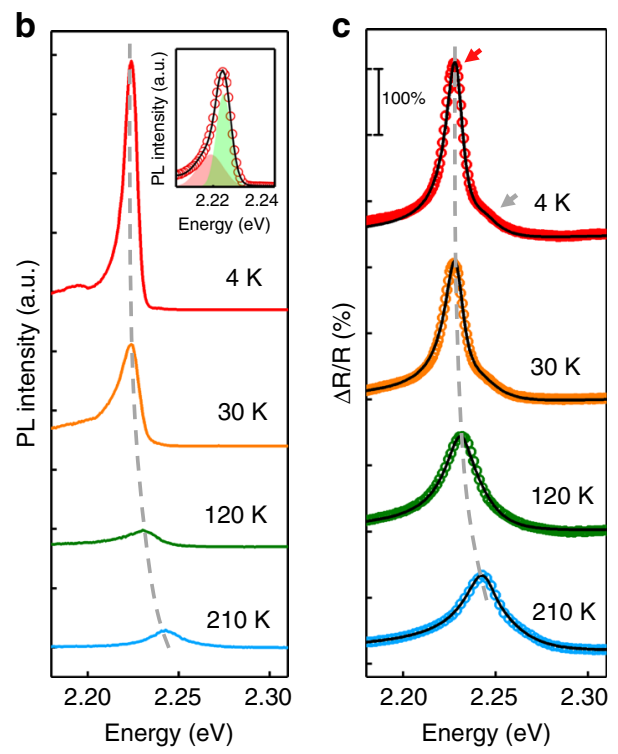

Fig. 3 Temperature-dependent absorption and PL of ML Me-PTCDI. a Normalized PL and differential reflectance spectrum of a ML Me-PTCDI sample at $4 \mathrm{~K}$. Inset: Polarization-dependent PL intensity. b Close-up temperature-dependent PL spectra of ML Me-PTCDI near the main peak. The gray dashed line shows the bathochromic shift of PL peak. c, $\mathbf{d}$ Close-up temperature-dependent differential reflectance spectra of $\mathrm{ML} \mathrm{Me-PTCDI} \mathrm{on} \mathrm{quartz} \mathrm{(c)} \mathrm{and} \mathrm{SiO} 2 / \mathrm{Si}$ (d). The red and gray arrows represent the differential reflectance from superradient state and single-molecule FE, respectively. e Temperature-dependent

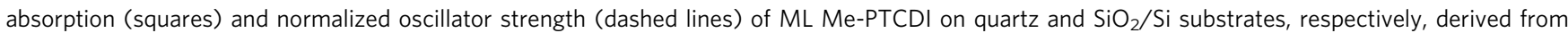
transfer matrix method.

of metal-sulfides to near unity, it rolls off below $1 \%$ at high excitation density that is most relevant to devices ${ }^{22,26}$. To demonstrate the bright PL from ML Me-PTCDI, we directly compared with several TMDs $\left(\mathrm{MoS}_{2}, \mathrm{WS}_{2}, \mathrm{MoSe}_{2}, \mathrm{WSe}_{2}\right)$ under $450 \mathrm{~nm}$ and $532 \mathrm{~nm}$ excitations. In Fig. 2c, while we observed bright luminescence from Me-PTCDI, the $\mathrm{MoSe}_{2}$ was nearly invisible. Quantitatively, the PL of ML Me-PTCDI was 2-5 orders of magnitude brighter than these TMDs under the same experimental conditions (Supplementary Fig. 6). We note that under non-resonant $450 \mathrm{~nm}$ and $532 \mathrm{~nm}$ excitations, the absorption of ML Me-PTCDI is lower than the TMDs ${ }^{26}$ (Fig. 3). Therefore, the much brighter PL from ML Me-PTCDI is due to enhanced PLQY.

\section{Temperature-dependent optical properties}

To further study the exciton properties in ML perylene derivatives, we performed temperature-dependent optical measurements. Figure 3a shows the PL and differential reflectance $(\Delta R / R)$ spectrum of ML Me-PTCDI at $4 \mathrm{~K}$ (the same sample, on quartz substrate). The PL showed drastically reduced linewidth of 8.5 $\mathrm{meV}$, little vibronic progression and nearly perfect linear polarization $\left(P=\frac{I_{\max }-I_{\min }}{I_{\max }+I_{\min }}=94.1 \%\right)$, due to increased $N_{\mathrm{c}}$ at low temperature. The PL could be fitted with two Gaussians (separated by $5.2 \mathrm{meV}$, attributed to Davydov doublet from the two molecules in the unit cell ${ }^{28}$, Fig. $3 \mathrm{~b}$ inset), indicating that the linewidth was inhomogeneously broadened due to the large number of molecules under laser spot. Interestingly, the absorption was also dominated by the same peak without Stokes shift (Fig. 3a). This means that the exciton gains very large oscillators strength such that it also dominates the absorption process. Aside from the main resonance peak, single-molecule FE also contributed to a small part of absorption as shown by the small peak on the high-energy shoulder (gray arrows in Fig. 3c, d) and its vibronic peak near $2.4 \mathrm{eV}$ (arrow in Fig. 3a, Supplementary Fig. 7).

Quantitative absorption and oscillator strength of ML MePTCDI was obtained by analyzing the temperature-dependent differential reflectance on both quartz (Fig. 3c) and $275 \mathrm{~nm}$
$\mathrm{SiO}_{2} / \mathrm{Si}$ substrates (Fig. 3d) using transfer matrix method ${ }^{29}$ (see "Methods" section). We also obtained the absorption by measuring transmittance and reflectance of ML Me-PTCDI on quartz substrate at room temperature (supplementary Fig. 7). Strong light-matter interaction is evidenced by the large $\Delta R / R$ amplitude at resonance. Our modeling using two oscillators (corresponding to the superradient and single-molecule state, respectively) was in excellent agreement with experimental data. The derived peak absorption at the main resonance consistently exceeds $30 \%$ at $4 \mathrm{~K}$ (Fig. 3e, the variation is due to different as-grown sample quality), which is among the highest of any ML materials ${ }^{30}$. Importantly, the absorption is not constant but became progressively stronger at low temperature, which is a direct proof of enhanced oscillator strength $f$ (Fig. 3e). Since we did not observe any sign of CT states from absorption ${ }^{31}$, it could only be explained by increasing exciton size in the J-aggregate. On the other hand, the single-molecule oscillator strength was much smaller than that of the main resonance (Supplementary Table 2). Further modeling on $\Delta \mathrm{R} / \mathrm{R}$ of multi-layer Me-PTCDI showed that the oscillator strength of the FE was about one order of magnitude smaller than ML (Supplementary Fig. 7, Supplementary Table 2). This is not surprising because the FE in multi-layers is heavily mixed with interlayer CT, leading to spatial separation of electrons and holes ${ }^{28,32}$.

We observed several other spectral changes to qualitatively support superradiance in ML. First, the exciton energy showed bathochromic shift on the order of $J$ and saturated at low temperature (Fig. 3b-d). This behavior is consistent with the theory of $1 \mathrm{D}$ J-aggregate ${ }^{33}$, where energy shift is described by $2 J \cdot \cos (\pi /$ $\left.\left(N_{c}+1\right)\right)$ and approaches asymptotically to $2 J$ for large $N_{c}$. We can rule out thermally induced strain or screening effects because the shift was not observed in multi-layers or monomers. Second, the PL exhibited strong increase of intensity and decrease of linewidth at low temperature (Fig. 3b), which was typical for superradiance ${ }^{34}$. The enhanced PL was also in line with the exciton oscillator strength from differential reflectance measurements. The brightening of PL was not observed in isolated monomers (Supplementary Fig. 8), indicating that coherent 
a

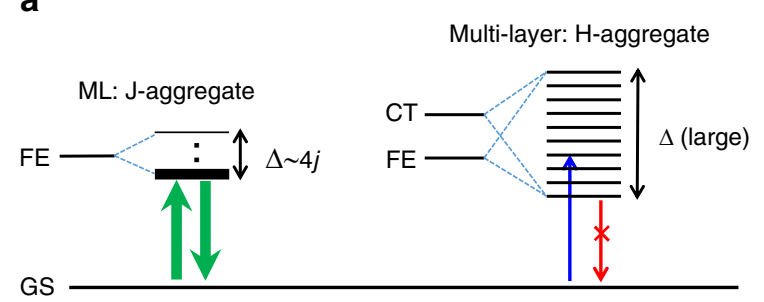

b

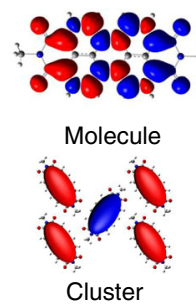

Multi-layer: $\mathrm{H}$-aggregate
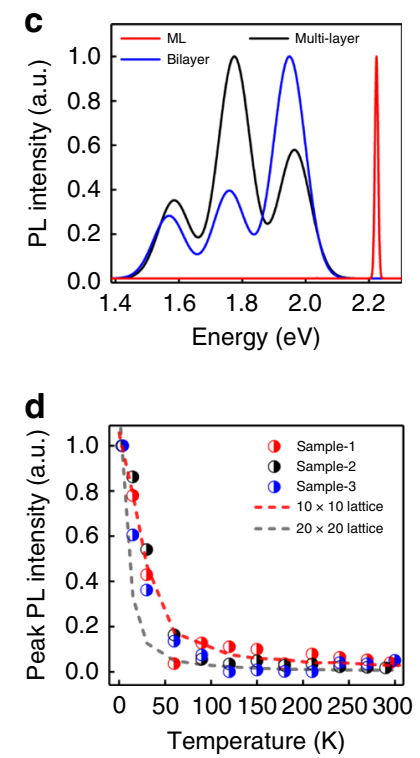

Fig. 4 Theoretical modeling of layer-dependent excitonic properties. a Schematic illustration of the excitonic band diagram of ML and multi-layer MePTCDI. For ML, the lowest excited state is purely Frenkel and coherent. For bulk, the excitonic states are mixed Frenkel/CT and incoherent. b Frenkel

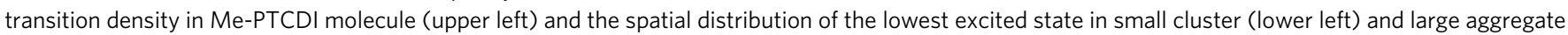
(right) in ML. Red and blue in single molecule denote transition electrons and holes. The color scale in cluster/aggregate stands for the value of exciton wavefunction at each site. c Calculated PL spectra of ML, bi-layer and multi-layer Me-PTCDI crystals. d Simulated temperature dependence of the PL emission intensity for ML (using $10 \times 10$ and $20 \times 20$ lattice) with comparisons to three experimental data sets.

dipole interaction was responsible. We note that near-unity PLQY at room temperature is expected to maintain at low temperature, because the increased coherent length would lead to even shorter radiative lifetime. To validate this hypothesis, we plot integrated PL and absorption at 532/514 $\mathrm{nm}$ as a function of temperature (Supplementary Fig. 5c, d). As expected, they show the same scaling trend with temperature, indicating that the PLQY remains relatively constant in the whole temperature range.

\section{Theoretical modeling}

We rationalized our experimental results theoretically using a Hamiltonian including Frenkel, CT and their coupling with parameters derived from first-principle calculations ${ }^{35,36}$ (see Methods). As illustrated in Fig. 4a, the excitations in multi-layer perylene derivatives are mixtures of Frenkel and CT states due to the large interlayer electronic coupling ( $D_{\mathrm{e}}$ for electrons, $D_{\mathrm{h}}$ for holes, Supplementary Table 1). The percentage of CT configurations is over $40 \%$ in bi-layer and quickly increased to the bulk value of $\sim 75 \%$ at 6 -layer (Supplementary Fig. 9c). On the other hand, the CT percentage in ML was negligible because the intralayer $D_{\mathrm{e}}$ and $D_{\mathrm{h}}$ were 1-2 orders of magnitude smaller, and the intralayer CT processes were 2 orders of magnitude slower (Supplementary Table 1, Supplementary Table 3). Indeed, we did not observe any evidence of CT states in ML absorption (Fig. 3a) ${ }^{31}$. As a result, the excitations in ML form a pure Frenkel J-band co mpletely separated from CT (Fig. 4a), which is a rare occasion in molecular systems ${ }^{36}$. Figure $4 \mathrm{~b}$ plots the spatial distribution of the lowest excitation in ML Me-PTCDI, showing a delocalized envelop wavefunction. The coherent interaction of many Frenkel dipoles forms a giant superradiant transition dipole. The ellipsoidal shape of the wavefunction also explains the linear PL anisotropy in experiment (Fig. 3a).

Within above theoretical framework, the calculated PL spectra successfully reproduced all the main experimental features (Fig. 4c, Supplementary Fig. 9). The narrow PL emission and supressed vibronic progressions in $\mathrm{ML}$ were only possible to reproduce with extended coherence and without CT (Supplementary Fig. 9d-f). The coherent length could be estimated by fitting the temperature-dependent PL intensity, which is widely adopted in the community ${ }^{37}$. We find that a lattice size of 10-20 $\left(N_{c} \sim 50-200\right)$ gives reasonably good agreement with experimental data (Fig. 4d, Supplementary Note 2), suggesting that $N_{c}$ could reach the order of 100 at zero temperature. Compared to most biological aggregates and molecular crystals ${ }^{13}$, the coherence in ML Me-PTCDI is much extended both spatially and temporally ( $\sim 30$ ps versus sub-picosecond). This unique property is only accessible at 2D limit owing to reduced screening and suppression of CT.

\section{Light-emitting devices}

The fact that $J$ is comparable to $k_{\mathrm{B}} T$ at room temperature has important implications on device applications. To demonstrate this aspect, we fabricated electrically injected light-emitting devices on ML perylene derivatives (in this case PTCDA). To get carrier injection into ML PTCDA on insulating h-BN, a oneterminal, alternating current (AC) driven vertical device structure was employed (Fig. 5a) ${ }^{38}$. In this device, ML PTCDA/h-BN on 90 $\mathrm{nm} \mathrm{SiO}_{2} / \mathrm{Si} \mathrm{p}^{++}$substrate was covered with few-layer CVD graphene as transparent source contact by micro-transferring, which was grounded through a metal bond pad (Fig. 5a). A ML CVD h-BN was inserted between graphene and PTCDA to prevent quenching of the EL. By applying an AC voltage to the $\mathrm{Si}$ backgate, alternating electrons and holes were injected into the ML both from the source contact and give transient electroluminescence $(t-E L)^{38}$. Figure $5 b$ shows the room-temperature $t$ EL spectrum under $20 \mathrm{~V}$ peak-to-peak square wave driving voltage. The peak at $2.224 \mathrm{eV}$ can be clearly identified, which overlaps with the PL of ML. The EL peak shows $\sim 50 \mathrm{meV}$ red shift and reduced linewidth by a factor of 2 compared to singlemolecule 0-0 Frenkel transition (at $2.274 \mathrm{eV}$ ). This suggests that the excitonic state can be excited electrically, which is an important step towards coherence-enabled device functions ${ }^{13}$. The EL intensity showed linear relationship with frequency as 


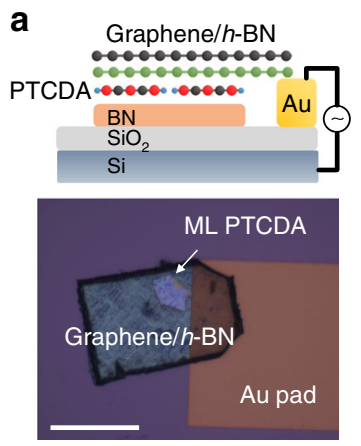

b

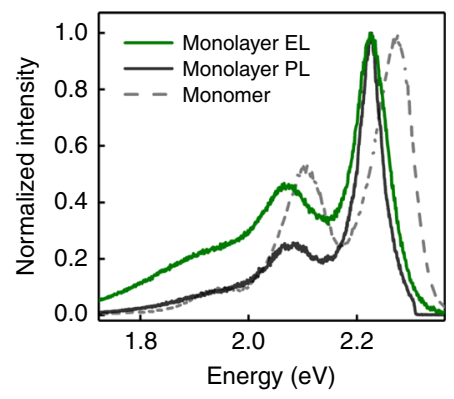

C

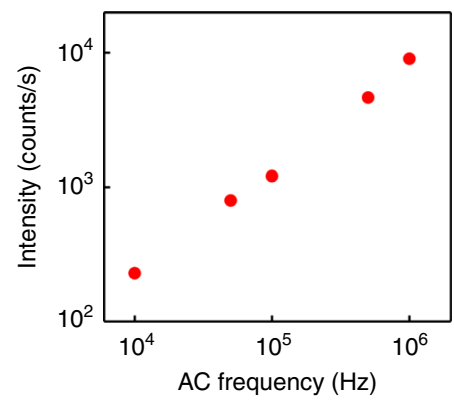

Fig. 5 Transient light-emitting devices of $\mathbf{M L}$ perylene derivatives. a Schematic and optical image of the ML PTCDA transient light-emitting device for electrically exciting the coherent state. Scale bar: $50 \mu \mathrm{m}$. b t-EL and PL spectra measured on the same ML PTCDA device. The EL is excited using $20 \mathrm{~V}$ peak-to-peak square wave driving voltage. c Frequency dependence of EL intensity.

expected for AC driven devices (Fig. $5 \mathrm{c})^{38}$. The linear trend persisted up to $20 \mathrm{MHz}$ without intensity drop (limited by the loss in our measurement circuit), indicating that our EL devices could operate at much higher frequency. Considering the roomtemperature PL lifetime $\sim 27 \mathrm{ps}$ (Fig. $2 \mathrm{~b}$ ), the intrinsic frequency limit should be above $30 \mathrm{GHz}$. Although the transient lightemitting devices are very preliminary, these results clearly demonstrate the potential of ML perylene derivatives for efficient and high-speed optoelectronic devices.

\section{Discussion}

In conclusion, we report unusual optical properties emerging from a class of ML molecular J-aggregate of perylene derivatives. The photo-excitation is dominated by FE with coherently delocalized wavefunction, due to large unscreened dipole interactions and nearly complete elimination of $\mathrm{CT}$ processes. As a result, ML Me-PTCDI shows resonant absorption over 30\%, bright PL emission (more than 2 orders of magnitude brighter than TMDs) and PLQY in the range of $60-100 \%$. The temperature dependence of oscillator strength, exciton energy and linewidth provide evidence of superradiance. In addition, we demonstrate light-emitting devices on ML perylene derivatives and show that the superradiance is robust under electrical injection. These strongly emitting ML molecular crystals can be integrated with other $2 \mathrm{D}$ materials to form van der Waals heterostructures on-demand ${ }^{39,40}$, opening many new opportunities in high-performance optoelectronics.

\section{Methods \\ vdW expitaxy growth of Me-PTCDI crystals on BN. We used mechanically exfoliated $\mathrm{BN}$ (hq graphene) on $275 \mathrm{~nm} \mathrm{SiO} / / \mathrm{Si}$ as the growth substrate without further cleaning. Before growth, the BN sheets were characterized by optical microscope and AFM to obtain the topological information. The growth of Me- PTCDI was carried out in a home-built tube furnace. The Me-PTCDI powder (Sigma Aldrich, 97\% without further purification) and substrates were placed at the center and downstream in the furnace, respectively. After evacuating the chamber to $\sim 3 \times 10^{-3} \mathrm{~Pa}$, the furnace was heated to $210-260^{\circ} \mathrm{C}$ to start growing Me-PTCDI crystals. The number of layers can be controlled by the heating temperature, growth duration and substrate position.}

AFM and Raman spectroscopy. AFM (both regular and high-resolution) was performed by Asylum Cypher under ambient condition. We used Asylum TR400PB tips for high-resolution AFM. Raman spectroscopy was performed by Princeton Instruments SP-2560 spectrometer coupled to a silicon CCD camera (Princeton Instruments PYL-1300BXD). We used a $488 \mathrm{~nm}$ laser as excitation.

TEM measurement. The selected-area electron diffraction (SAED) pattern was collected on a $200 \mathrm{kV}$ FEI Tecnai F20 transmission electron microscope (TEM). The TEM samples were prepared by transferring few-layer BN sheets exfoliated on $\mathrm{SiO}_{2} / \mathrm{Si}$ substrate onto $\mathrm{Cu}$ TEM grid with carbon film, using the process described in reference ${ }^{41}$ and growing organic films on transferred BN. As this organic crystal sample is highly electron beam sensitive, the SAED pattern was acquired with a short exposure time of $0.01 \mathrm{~s}$ to prevent from the beam damage. The SAED simulations were calculated using the multislice method with the Kirkland code ${ }^{42}$.

Optical spectroscopies. The PL images were collected using an optical microscope (Olympus BX51M). The samples were illuminated by a $450 \mathrm{~nm}$ LED and the PL images were collected by a colored CCD camera. The excitation light was filtered by a long-pass filter in front of the CCD.

Temperature-dependent PL measurements were done inside an optical cryostat (Montana Cryostation S50) coupled to Horiba LabRAM HR800 system. We used $532 \mathrm{~nm}$ laser as excitation. The base temperature of the cryostat was $4 \mathrm{~K}$. The PL were collected by a $50 \times$ objective $(\mathrm{NA}=0.45)$. Polarization-dependent PL was tested by rotating a linear polarizer between the sample and detector, while keeping the incident laser polarization.

For TRPL measurement, the excitation light is generated by a mode-locked $\mathrm{Ti}$ : sapphire laser with an optical parametric oscillator. The laser pulse width is on the order of $200 \mathrm{fs}$. The light is focused onto the sample by a Zeiss $50 \times$ objective. The emission signal, detected in the reflection configuration, is passed through a bandpass filter with a bandwidth of $20 \mathrm{~nm}$ and collected by a synchroscan Hamamatsu streak camera (C10910-02), whose overall time resolution is 2 ps.

For absorption measurements, the sample was placed in cryostat chamber (OXFORD INSTRUMENTS_MicrostatHires) cooled by liquid helium. The broadband radiation from a halogen lamp covering the spectral range from 450 to $900 \mathrm{~nm}$ was radiated on the sample without focus. The transmission signal was collected by $50 \times$ objective $(\mathrm{NA}=0.7)$ and analyzed by a liquid nitrogen cooled silicon CCD detector with a spectrograph. Absorption signal was determined by measured normalizing the transmission signal from the sample on BN/fused silica to that from the bare fused silica.

PLQY measurement. We use a dedicated setup at UC Berkeley to measure PLQY. The same instruments and procedures were used as in several previously papers ${ }^{22,43}$. Below we describe the details of the measurement (Supplementary Fig. 5a). As excitation source, an $\mathrm{Ar}+$ laser (Lexel 95) with $514 \mathrm{~nm}$ line was utilized in steady-state PL and the power density was adjusted by neutral density filters and simultaneously monitored by photodiode power sensor (ThorLabs S120C). The ratio of laser power on the diode to incident power onto sample was around 50 so that the low laser power can be accurately measured. A Si CCD detector (Andor iDus BEX2-DD) on an $f=340 \mathrm{~mm}$ spectrometer with a $150 \mathrm{~g} \mathrm{~mm}^{-1}$ grating was used to collect the steady-state PL spectra and the dark background of CCD was measured and subtracted each time from the acquired signal (Supplementary Fig. 5a, left). A $50 \times$ MD Plan (Olympus) objective lens (numerical aperture, 0.55) was used for all measurements. For PLQY and room temperature quantitative absorption measurements, we used quartz substrates to avoid optical interference from $\mathrm{Si} / \mathrm{SiO}_{2}$ substrates. Calibration for the external sample PL efficiency was performed using the wavelength-dependent instrument function which characterized the collection efficiency of the instrument. This calibration process was previously described in our work and have below $15 \%$ error when cross calibrated with a Si photodiode or using a dye solution of unity quantum yield ${ }^{22}$. In brief, the instrument function is measured using a near ideal diffuse reflector as the source of calibration. The internal PLQY was extracted from the measured external quantum efficiency using the quantitative absorption at the pump wavelength and by the fraction of light within escape cone $\left(1 / 4 n^{2}\right.$, where $n$ is the refractive index of the medium).

To quantitatively calculate PLQY, we need to measure the absolute absorption of ML Me-PTCDI at $514 \mathrm{~nm}$. Here the absorption is measured by $100 \%$-T-R, where the transmittance and reflectance are individually determined using lock-in detection (Supplementary Fig. 5a, right). The laser was focused on the sample using a $50 \times$ objective, the reflected light was collected via the same objective and the 
transmitted light was collected by a $20 \times$ objective. The system was calibrated using quartz and silver as reference transmission and reflectance standards. The reported generation rates (steady-state measurements) and initial carrier densities (timeresolved measurements) are calculated from the number of incident photons per unit area and the absorption.

ACEL device. The ACEL device of PTCDA is fabricated by multiple transfer steps, where one layer of graphene and one layer of h-BN is put on top of the ML molecular crystal. Specifically, the device was fabricated by two methods and gave similar result, indicating the molecular monolayer can survive the transfer process.

Method 1: An array of Au pads $(75 \mu \mathrm{m} \times 75 \mu \mathrm{m}$ square $)$ is fabricated on $90 \mathrm{~nm}$ $\mathrm{SiO}_{2} / \mathrm{Si} \mathrm{p}++$ substrates by photolithography. Using PMMA pad $(\sim 400 \times 400 \mu \mathrm{m})$ to sequentially pick up CVD multi-layer graphene (on $\mathrm{Si} / \mathrm{SiO}_{2}$ substrate), $\mathrm{CVD}$ monolayer h-BN (on $\mathrm{Si} / \mathrm{SiO}_{2}$ substrate), and the $\mathrm{ML}$ on $\mathrm{BN}$, and put down the stack on pre-patterned substrate (metal electrode on $\mathrm{Si}_{2} \mathrm{SiO}_{2}$ ) so that the top graphene layer overlap with both the metal pad electrode and the ML. In a typical pick up process, the PMMA is first put on top of the target layer (graphene, $\mathrm{BN}$, or ML on BN) by a micro-tip, and the substrate is heated at $180^{\circ} \mathrm{C}$ for $90 \mathrm{~s}$ so that the PMMA would pass the glass transition temperature and bound to the target layer. Then an engraver and a micro-tip is used to decouple the PMMA from the substrate with the target layer and transfer the PMMA stack to another target layer. In this process the ML layer survived $180^{\circ} \mathrm{C}$ heating during the dry transfer.

Method 2: To reduce sequential dry transfers and increase device fabrication yield, the same dry transfer processed was used to first pick up a monolayer CVD h-BN, put down on ML-BN, then pick up this stack and transfer to the patterned substrate. Then dichloromethane is used to dissolve the PMMA of this stack, and another PMMA is used to pick up the graphene contact and put down on this substrate so that the graphene covers the electrode and the CVD BN-ML-BN stack. The device fabricated in this method behave similarly to method 1 , and in this process the heterostructure survives the dichloromethane solvent treatment.

In this device, the graphene on top bridge the Au pad and the PTCDA and allows carrier injection into the PTCDA ML. The CVD monolayer h-BN and CVD multilayer graphene was purchased from Graphene Supermarket.

Theoretical model and derivation of the Hamiltonian parameters. To qualitatively describe the nature of the excitonic states in Me-PTCDI, we employ the widely used electronic-vibrational model for molecular excitonic systems $\mathrm{s}^{35}$, in which the electronic part is

$$
\begin{aligned}
H_{\mathrm{el}}= & \sum_{m} \omega_{\mathrm{FE}}|m\rangle\left\langle m\left|+\sum_{\langle m n\rangle} J\right| m\right\rangle\left\langle n\left|+\sum_{n_{+}, n_{-}} \omega_{\mathrm{CT}}\right| n_{+} ; n_{-}\right\rangle\left\langle n_{+} ; n_{-}\right| \\
& +\sum_{n_{+},\left\langle n_{-}, n_{-}^{\prime}\right\rangle} t_{\mathrm{e}}\left|n_{+} ; n_{-}\right\rangle\left\langle n_{+} ; n_{-}^{\prime}\left|+\sum_{\left\langle n_{+}, n_{+}^{\prime}, n_{-}\right\rangle} t_{\mathrm{h}}\right| n_{+} ; n_{-}\right\rangle\left\langle n_{+}^{\prime} ; n_{-}\right| \\
& +\sum_{\langle m n\rangle} D_{\mathrm{e}}(|n\rangle\langle n ; m|+\text { h.c. })+\sum_{\langle m n\rangle} D_{\mathrm{h}}(|n\rangle\langle m ; m|+\text { h.c. }),
\end{aligned}
$$

where the first two terms represent Frenkel states, the next three terms represent CT states, and the last two terms are the coupling between them. The excited eigenstates are linear combinations of the FE and CT configurations, and the coefficients depends on their energy difference $\left(\Delta_{\mathrm{FE}-\mathrm{CT}}=\left|\omega_{\mathrm{FE}}-\omega_{\mathrm{CT}}\right|\right)$ as well as the off-diagonal coupling terms ( $J$ for interaction between FE ones, $t_{\mathrm{e}}$ and $t_{\mathrm{h}}$ for charge hopping integral among CT ones, and $D_{\mathrm{e}}$ and $D_{\mathrm{h}}$ for coupling between FE and CT states).

The vibrational part is

$$
\begin{aligned}
H_{\text {vib }}= & \omega_{\text {vib }} \sum_{n} b_{n}^{\dagger} b_{n}+\omega_{\text {vib }} \sum_{n}\left\{\lambda\left(b_{n}^{\dagger}+b_{n}\right)+\lambda^{2}\right\}|n\rangle\langle n| \\
& +\omega_{\text {vib }} \sum_{n_{+}, n_{-}}\left\{\lambda_{+}\left(b_{n_{+}}^{\dagger}+b_{n_{+}}\right)+\lambda_{-}\left(b_{n_{-}}^{\dagger}+b_{n_{-}}\right)+\lambda_{+}^{2}+\lambda_{-}^{2}\right\}\left|n_{+} ; n_{-}\right\rangle\left\langle n_{+} ; n_{-}\right|
\end{aligned}
$$

The first term is the pure vibrational energy. And $b_{n}^{\dagger}$ creates a vibrational excitation with energy $\omega_{\mathrm{vib}}$ on site $n$, whereas $b_{n}$ annihilates the same. The last two terms are the electron-phonon coupling, and $\lambda^{2}, \lambda_{+}^{2}$ and $\lambda_{-}^{2}$ represent Huang-Rhys factors $^{44}$ for exciton, cation and anion respectively.

The Hamiltonian parameters shown in Supplementary Table 1 are derived from DFT and time-dependent density functional theory (TDDFT) calculations of molecular monomers or dimers at M06-2×/6-31G level by using GAUSSIAN 09 program ${ }^{45}$.

$\omega_{0-0}$ and $\omega_{\text {vib }}$ are extracted from the vibronic spectra calculations by using the Franck-Condon method after a TDDFT calculation for the pure electronic excitation for the molecular monomer. $\lambda^{2}, \lambda_{+}^{2}$ and $\lambda_{-}^{2}$ are calculated by using Duschinsky rotation method with DUSHIN program ${ }^{46}$. The excitonic couplings $J$ are calculated using the Generalized Mulliken-Hush (GMH) method ${ }^{47}$. The charge-transfer integrals $\left(D_{\mathrm{e}}\right.$ and $\left.D_{\mathrm{h}}\right)$ are computed as the Fock matrix elements between frontier molecular orbitals of the neighboring molecules.

For computing the calculated time scale for inter-molecular electron/hole charge transfer in Me-PTCDI crystals, we use the Marcus theory to calculate the intermolecular CT rate $(k)$

$$
\frac{1}{t}=k=\frac{2 \pi}{\hbar} \frac{\left|H_{\mathrm{ab}}\right|^{2}}{\sqrt{4 \pi \lambda k_{\mathrm{B}} T}} \exp \left[-\frac{(\lambda+\Delta G)^{2}}{4 \lambda k_{\mathrm{B}} T}\right] .
$$

Here $T$ is the temperature, $k_{\mathrm{B}}$ is the Boltzmann constant, $H_{\mathrm{ab}}$ is the electronic coupling $\left(D_{\mathrm{e}}\right.$ and $D_{\mathrm{h}}$ for electron and hole respectively), $\lambda$ is the reorganization energy and $\Delta G$ is free energy (in this case, we use $\Delta G=0$ ). We calculated the reorganization energy values for the electron $\left(\lambda_{-}\right)$and hole $\left(\lambda_{+}\right)$carriers using the following equation:

$$
\lambda_{-/+}=\left(E_{-/+}^{*}-E_{-/+}\right)+\left(E_{-/+}^{* *}-E_{0}\right)
$$

where $E_{-t_{+}}^{*}$ is the vertical energy of negatively/positively charged molecule at the geometry of the optimized neutral molecules, $E_{-/+}$is the optimized energy of negatively/positively charged molecules, $E_{-/+}^{* *}$ is the neutral energy of molecules at the geometry of optimized negatively/positively charged species, and $E_{0}$ is the ground-state energy at the optimized geometry of the neutral molecule.

Transfer matrix method of calculating absorption. We model the dielectric function of Me-PTCDI by the Lorentz model. The dielectric function is given by

$$
\epsilon(E)=\epsilon_{\infty}+\sum_{i=1}^{N} \frac{f_{i} E_{i}^{2}}{E_{i}^{2}-E^{2}+i \gamma_{i} E}
$$

where $E_{i}, \gamma_{i}$ and $f_{i}$ are the resonance energy, damping rate and oscillator strength of the $i^{t h}$ exciton, respectively. $\epsilon_{\infty}$ is the background dielectric constant that accounts for the contributions from resonances at higher energies.

Given the dielectric function of Me-PTCDI, the differential reflectance spectra then can be directly calculated using the transfer matrix method. Here we use the $\mathrm{BN} /$ quartz substrate as an example. In general, the transfer matrix at a dielectric boundary between two media of refractive indices $n_{1}$ and $n_{2}$ are given by

$$
M_{12}=\frac{1}{n_{1}+n_{2}}\left(\begin{array}{ll}
n_{2}+n_{1} & n_{2}-n_{1} \\
n_{2}-n_{1} & n_{2}+n_{1}
\end{array}\right)
$$

And the propagation matrix within a uniform layer of refractive index $n_{l}$ and thickness $d_{l}$ is given by

$$
P_{l}=\left(\begin{array}{cc}
e^{i n_{l} k d_{l}} & 0 \\
0 & e^{-i n_{l} k d_{l}}
\end{array}\right)
$$

where $k$ is the wave vector in free space.

The transfer matrices for the Me-PTCDI layer on the substrate, and the pure substrate then can be written as

$$
\begin{gathered}
M_{\mathrm{P}}=M_{\text {air }, \mathrm{p}} P_{\mathrm{PTCDI}} M_{\mathrm{p}, \mathrm{BN}} P_{\mathrm{BN}} M_{\mathrm{BN}, \mathrm{q}}=\left(\begin{array}{cc}
1 / t_{\mathrm{p}} & r_{\mathrm{p}}^{*} / t_{\mathrm{p}}^{*} \\
r_{\mathrm{p}} / t_{\mathrm{p}} & 1 / t_{\mathrm{p}}^{*}
\end{array}\right) \\
M_{\text {sub }}=M_{\text {air }, \mathrm{BN}} P_{\mathrm{BN}} M_{\mathrm{BN}, \mathrm{q}}=\left(\begin{array}{cc}
1 / t_{\mathrm{sub}} & r_{\mathrm{sub}}^{*} / t_{\mathrm{sub}}^{*} \\
r_{\mathrm{sub}} / t_{\mathrm{sub}} & 1 / t_{\mathrm{sub}}^{*}
\end{array}\right)
\end{gathered}
$$

The differential spectrum $\Delta R / R$ then is given by

$$
\frac{\Delta R}{R}=\frac{\left|r_{\mathrm{p}}\right|^{2}-\left|r_{\text {sub }}\right|^{2}}{\left|r_{\text {sub }}\right|^{2}}
$$

The dielectric function of Me-PTCDI then can be obtained by fitting the differential reflectance spectrum.

For all the samples, the thickness of the $\mathrm{h}-\mathrm{BN}$ was measured by AFM and accounted for in the modeling. In addition, the reference point was measured on the same $\mathrm{BN}$. This was achieved by covering part of the $\mathrm{BN}$ by Au pad and peeling it off after Me-PTCDI

\section{Data availability}

The data that support the findings of this study are available from the corresponding author upon reasonable request.

Received: 17 September 2019; Accepted: 12 November 2019; Published online: 06 December 2019

\section{References}

1. Xia, F., Wang, H., Xiao, D., Dubey, M. \& Ramasubramaniam, A. Twodimensional material nanophotonics. Nat. Photon. 8, 899-907 (2014).

2. Schaibley, J. R. et al. Valleytronics in 2D materials. Nat. Rev. Mater. 1, 16055 (2016).

3. Mak, K. F. \& Shan, J. Photonics and optoelectronics of 2D semiconductor transition metal dichalcogenides. Nat. Photon. 10, 216-226 (2016). 
4. You, Y. et al. Observation of biexcitons in monolayer WSe2. Nat. Phys. 11, 477-481 (2015).

5. Hu, Z. et al. Impact of backbone fluorination on nanoscale morphology and excitonic coupling in polythiophenes. Proc. Natl Acad. Sci. USA 114, 5113 (2017).

6. Yang, F. et al. 2D organic materials for optoelectronic applications. $A d v$. Mater. 30, 1702415 (2018).

7. Jiang, L. et al. Millimeter-sized molecular monolayer two-dimensional crystals. Adv. Mater. 23, 2059-2063 (2011).

8. Wang, C. et al. W. Organic single crystals: N-Type 2D ORganic Single Crystals for High-performance Organic Field-effect Transistors and Near-infrared Phototransistors. Adv. Mater. 30, 1870114 (2018).

9. Shi, Y. et al. Bottom-up growth of n-type monolayer molecular crystals on polymeric substrate for optoelectronic device applications. Nat. Commun. 9, 2933 (2018).

10. Gobbi, M., Orgiu, E. \& Samorì, P. When 2D materials meet moleculesopportunities and challenges of hybrid organic/inorganic van der waals heterostructures. Adv. Mater. 30, 1706103 (2018).

11. Jelley, E. E. Spectral absorption and fluorescence of dyes in the molecular state. Nature 138, 1009-1010 (1936).

12. Kobayashi, T. J-Aggregates Vol. 2 (Word Scientific, Press, Singapore, 2012).

13. Scholes, G. D. et al. Using coherence to enhance function in chemical and biophysical systems. Nature 543, 647-656 (2017).

14. Ponseca, C. S. Jr, Chábera, P., Uhlig, J., Persson, P. \& Sundström, V. Ultrafast electron dynamics in solar energy conversion. Chem. Rev. 117, 10940-11024 (2017).

15. Pšenčík, J. et al. Lamellar organization of pigments in chlorosomes, the light harvesting complexes of green photosynthetic bacteria. Biophys. J. 87, 1165-1172 (2004).

16. He, D. et al. Two-dimensional quasi-freestanding molecular crystals for highperformance organic field-effect transistors. Nat. Commun. 5, 5162 (2014).

17. Zhang, Y. et al. Probing carrier transport and structure-property relationship of highly ordered organic semiconductors at the two-dimensional limit. Phys. Rev. Lett. 116, 016602 (2016).

18. Wu., B. et al. Precise, self-limited epitaxy of ultrathin organic semiconductors and heterojunctions tailored by van der waals interactions. Nano. Lett. 16, 3754-3759 (2016).

19. Wang, Q. H. \& Hersam, M. C. Room-temperature molecular-resolution characterization of self-assembled organic monolayers on epitaxial grapheme. Nat. Chem. 1, 206-211 (2009).

20. Zhao, Y. \& Wang, J. How to obtain high-quality and high-stability interfacial organic layer: insights from the PTCDA self-assembly. J. Phys. Chem. C 121, 4488-4495 (2017).

21. Forrest, S. R. Ultrathin Organic films grown by organic molecular beam deposition and related techniques. Chem. Rev. 97, 1793-1896 (1997).

22. Amani, M. et al. Near-unity photoluminescence quantum yield in $\mathrm{MoS}_{2}$. Science 350, 1065-1068 (2015)

23. Nollau, A., Hoffmann, M., Floreck, K., Fritz, T. \& Leo, K. A simple measurement of the absolute internal quantum efficiency of thin organic films. J. Appl. Phys. 87, 7802 (2000).

24. Bigeon, J. et al. Strong coupling between self-assembled molecules and surface plasmon polaritons. J. Phys. Chem. Lett. 8, 5626-5632 (2017).

25. Xu, R. et al. Exciton brightening in monolayer phosphorene via dimensionality modification. Adv. Mater. 28, 3493-3498 (2016).

26. Amani, M. et al. Recombination kinetics and effects of superacid treatment in sulfur- and selenium-based transition metal dichalcogenides. Nano. Lett. 16, 2786-2791 (2016).

27. Withers, F. et al. Light-emitting diodes by band-structure engineering in van der Waals heterostructures. Nat. Mater. 14, 301-306 (2015).

28. Hoffmann, M. et al. The lowest energy Frenkel and charge-transfer excitons in quasi-one-dimensional structures: application to MePTCDI and PTCDA crystals. Chem. Phys. 258, 73-96 (2000).

29. Bahaa, E. A., Saleh, B. E. A. \& Teich, M. C. Fundamentals of Photonics. (John Wiley \& Sons, Inc., Press, USA, 1991).

30. Li, Y. Measurement of the optical dielectric function of monolayer transitionmetal dichalcogenides: MoS2, MoSe2, WS2, and WSe2. Phys. Rev. B 90, 205422 (2014).

31. Proehl, H., Dienel, T., Nitsche, R. \& Fritz, T. Formation of solid-state excitons in ultrathin crystalline films of PTCDA: from single molecules to molecular stacks. Phys. Rev. Lett. 93, 097403 (2004).

32. Ma, H. \& Troisi, A. Direct optical generation of long-range charge-transfer states in organic photovoltaics. Adv. Mater. 26, 6163-6167 (2014).

33. Hestand, N. J. \& Spano, F. C. Expanded Theory of H- and J-Molecular Aggregates: The Effects of Vibronic Coupling and Intermolecular Charge Transfer. Chem. Rev. 118, 7069-7163 (2018).

34. Cong, K. et al. Dicke superradiance in solids. J. Opt. Soc. Am. B: Opt. Phys. 33, C80-C101 (2016).
35. Yamagata, H. et al. HJ-aggregate behavior of crystalline 7,8,15,16tetraazaterrylene: introducing a new design paradigm for organic materials. J. Phys. Chem. C. 118, 28842-28854 (2014).

36. Bardeen, C. J. The structure and dynamics of molecular excitons. Annu. Rev. Phys. Chem. 65, 127-148 (2014)

37. Lim, S. H., Bjorklund, T. G., Spano, F. C. \& Bardeen, C. J. Exciton delocalization and superradiance in tetracene thin films and nanoaggregates. Phys. Rev. Lett. 92, 107402 (2004).

38. Lien, D.-H. et al. Large-area and bright pulsed electroluminescence in monolayer semiconductors. Nat. Commun. 9, 1229 (2018).

39. Wang, S. et al. A MoS2/PTCDA hybrid heterojunction synapse with efficient photoelectric dual modulation and versatility. Adv. Mater. 31, 1806227 (2018)

40. Chen, $\mathrm{X}$. et al. Improving the performance of graphene phototransistors using a heterostructure as light-absorbing layer. Nano Lett. 17, 6391 (2017).

41. Sundar, V. C. et al. Elastomeric transistor stamps: reversible probing of charge transport in organic crystals. Science 303, 1644 (2004).

42. Kirkland, E. J. Advanced Computing in Electron Microscopy (Springer, 2010)

43. Lien, D.-H. et al. Electrical suppression of all nonradiative recombination pathways in monolayer semiconductors. Science 364, 468-471 (2019).

44. Motta, S. D. et al. Resistive molecular memories: influence of molecular parameters on the electrical bistability. J. Am. Chem. Soc. 131, 6591-6598 (2009).

45. Frisch, M. J. et al. Gaussian 09, Revision D.01 (Gaussian, Inc., 2009).

46. Jeffrey, R. Reimers A practical method for the use of curvilinear coordinates in calculations of normal-mode-projected displacements and Duschinsky rotation matrices for large molecules. J. Chem. Phys. 115, 9103 (2001).

47. Hsu, C.-P. The electronic couplings in electron transfer and excitation energy transfer. Acc. Chem. Res. 42, 509-518 (2009).

\section{Acknowledgements}

We acknowledge stimulating discussions with Xiaomu Wang, Yuerui Lu and Zongfu Yu. The work at Nanjing University is supported by National Natural Science Foundation of China 61734003, 61521001, 61851401, 51861145202, 61861166001, 21722302, 11874199; National Key Basic Research Program of China 2015CB921600; Strategic Priority Research Program of Chinese Academy of Sciences XDB 30000000; Key Laboratory of Advanced Photonic and Electronic Materials, Collaborative Innovation Center of SolidState Lighting and Energy-Saving Electronics, and the Fundamental Research Funds for the Central Universities of China. The work at Southeast University is supported by National Key Research and Development of China 2017YFA0205700. The work at Chinese University of Hong Kong is supported by RGC of Hong Kong SAR under AoE/ P-03/08, CUHK Group Research Scheme. The optical measurement in Berkeley is supported by the U.S. Department of Energy, Office of Science, Office of Basic Energy Sciences, Materials Sciences and Engineering Division, under contract no. DE-AC0205CH11231 within the Electronic Materials Program (KC1201).

\section{Author contributions}

X.W. conceived and supervised the project; H.Z., W.L., L.T., Y.M. J.Z., and X.C. prepared and characterized the samples; H.Z. and Z.W. performed PL experiments and data analysis; H.Z. performed absorption experiments and data analysis; Z.N., Y. Shi, J.X., and X.W. contributed to the analysis of PL and absorption data; Y.Z., J.X., Y.W., D.L., M.A., H.K., X.Z., and A.J. performed PLQY and lifetime experiments and data analysis. Y. Song, H.M., and A.T. performed theoretical calculations; M.Z. performed transfer matrix modeling; Y. F., B.S., and P.W. performed TEM and data analysis. H.Z., Y.Z., Y. Song., H.M., A.J., and X.W. co-wrote the manuscript with input from other authors. All authors contributed to discussions.

\section{Competing interests}

The authors declare no competing interests.

\section{Additional information}

Supplementary information is available for this paper at https://doi.org/10.1038/s41467 019-13581-9.

Correspondence and requests for materials should be addressed to H.M., A.J. or X.W.

Peer review information Nature Communications thanks Yanke Che and the other, anonymous, reviewer(s) for their contribution to the peer review of this work.

Reprints and permission information is available at http://www.nature.com/reprints

Publisher's note Springer Nature remains neutral with regard to jurisdictional claims in published maps and institutional affiliations. 
(c) Open Access This article is licensed under a Creative Commons Attribution 4.0 International License, which permits use, sharing, adaptation, distribution and reproduction in any medium or format, as long as you give appropriate credit to the original author(s) and the source, provide a link to the Creative Commons license, and indicate if changes were made. The images or other third party material in this article are included in the article's Creative Commons license, unless indicated otherwise in a credit line to the material. If material is not included in the article's Creative Commons license and your intended use is not permitted by statutory regulation or exceeds the permitted use, you will need to obtain permission directly from the copyright holder. To view a copy of this license, visit http://creativecommons.org/ licenses/by/4.0/.

(C) The Author(s) 2019 